\title{
Charge confinement and Klein tunneling from doping graphene
}

\author{
C. Popovici, ${ }^{1,2,3}$ O. Oliveira,,${ }^{1,2}$ W. de Paula, ${ }^{2}$ and T. Frederico ${ }^{2}$ \\ ${ }^{1}$ Departamento de Fúsica, Universidade de Coimbra, 3004-516 Coimbra, Portugal \\ ${ }^{2}$ Departamento de Física, Instituto Tecnológico de Aeronáutica, 12.228-900, São José dos Campos, SP, Brazil \\ ${ }^{3}$ Institut für Theoretische Physik, Universität Giessen, 35392 Giessen, Germany
}

\begin{abstract}
In the present work, we investigate how structural defects in graphene can change its transport properties. In particular, we show that breaking of the sublattice symmetry in a graphene monolayer overcomes the Klein effect, leading to confined states of massless Dirac fermions. Experimentally, this corresponds to chemical bonding of foreign atoms to carbon atoms, which attach themselves to preferential positions on one of the two sublattices. In addition, we consider the scattering off a tensor barrier, which describes the rotation of the honeycomb cells of a given region around an axis perpendicular to the graphene layer. We demonstrate that in this case the intervalley mixing between the Dirac points emerges, and that Klein tunneling occurs.
\end{abstract}

PACS numbers: 72.80.Vp, 71.10.-w, 68.55.Ln, 64.70.Nd

\section{INTRODUCTION AND MOTIVATION}

Graphene, a monatomic layer of carbon atoms on a honeycomb lattice, has been synthesized for the first time in $2004^{1,2}$. Its remarkable properties, such as unconventional quantum Hall effect $\underline{3,4}$, , Klein tunneling ${ }^{5}, \underline{6}$ or charge confinement ${ }^{7}$, are mainly a consequence of the fact that at low energies, the charge carriers in graphene are described by the relativistic Dirac equation instead of the more familiar Schrödinger equation $\underline{8,9}$. The single particle dispersion relation is linear in the momentum, $E_{k}= \pm v_{F}|\vec{k}|$, where $v_{F}$ is the Fermi velocity and $\vec{k}=\left(k_{x}, k_{y}\right)$ is the fermion momentum, measured relative to the inequivalent corners of the Brillouin zone $K$ and $K^{\prime}$, known as Dirac points. The peculiar properties of graphene triggered immediately a lot of interest, due to its possible applications in nanoscale devices, but also because graphene is able to connect different branches of physics. Meanwhile, we have learned how to produce multilayers of graphene, have started to understand the effects due to the deviations from a perfect carbon honeycomb structure and, most important from the point of view of technological applications, are learning on how to grow samples with structural defects. Defects can be used to tailor graphene based devices - see, for example, the discussions below. However, they can also change the transport properties of the charge carriers and be at the origin of the performance deterioration of these devices. Comprehensive overviews of graphene can be found in Refs. 10 14, and the structural defects are reviewed in $\underline{15}$. The work reported in $\underline{\underline{16}}$ gives an example of an experimental realization of one dimensional (1D) defects in pure graphene.

Typically, graphene based devices require the ability to confine and control the charge flow. Confining Dirac fermions is yet a challenging task, due to the so-called Klein effect $\underline{17} \underline{-19}$, where a relativistic electron is able to penetrate a barrier higher than the electron's energy completely unreflected - in contrast to the conventional tunneling where the transmission probability drops off exponentially as the barrier gets higher. Studies of various types of barriers that lead to Klein tunneling for electrons in graphene can be found in the literature, and experimental observations of this effect have been reported ${ }^{20}-25$. Despite the difficulties related to overcoming the Klein effect, charge confinement in graphene has already been accomplished experimentally — see for example Refs. 7, 10,26 for overviews. Theoretically, confinement was demonstrated by cutting or bending graphene sheets 27,28 , exploiting the transversal degrees of freedom of the electrons in an electrostatic potential 29 , applying magnetic fields $\frac{30,31}{31}$, deforming the graphene membranes 32 or by spatial modulation of the Dirac gap 33,34 . From structural defects, confinement has been achieved in graphene-graphane systems, see for example ${ }^{35}$. However, so far it has not been explored a way to produce charge confinement in association with chemical bonding of foreign atoms to carbon atoms, although techniques to implement the chemical bonding of some adsorbents, such as fluorine, hydrogen or oxygen, already exist $\underline{36}-38$.

In addition to the possible technological applications, the 1D defects in pristine graphene are interesting because they can be modeled as potential barriers, associated with different fermionic operators in the Dirac equation. Within the conceptual framework of effective quantum field theories, the authors of 39 generalize the results of 40,41 — where vortex formation in graphene is described through a chiral gauge theory - and introduce a scalar and a gauge field that account for the dynamics of the self-interaction of the carbon background and the mean self-interaction of the Dirac fermions. This model is well suited to describe various disorder phenomena such as topological defects, doping defects or distortions of the lattice honeycomb. Within this framework, carbon nanotubes and graphene have been studied and their quantum properties have been reproduced ${ }^{42,43}$.

In the present study, inspired by the work of $\underline{39-41}$, we explore how charge confinement and Klein tunneling can 
be induced by certain types of defects, and examine how defects - modeled as $1 \mathrm{D}$ potential barriers - can be mapped into fermionic operators. As described below, charge localization can be achieved via a barrier which breaks the sublattice symmetry. In practice, the breaking of the sublattice symmetry can be realized by binding covalently foreign atoms with particular carbons, whereas for the theoretical analysis, we have to identify and explore the corresponding fermionic operators. The Klein effect is investigated by considering the scattering of an electron off a tensor barrier. The associated fermionic operator generates a rotation around the $z$-axis (perpendicular on the graphene plane) and it couples both the two sublattices $A$ and $B$, and the two valleys $K$ and $K^{\prime}$. Experimentally this can be implemented by topologically distorting the graphene layer via a rotation of the honeycomb cells around the $z$-axis in a particular region, relative to the remaining graphene sheet. In order to include the effects of the $K \leftrightarrow K^{\prime}$ mixing, we work in the four-component, as opposed to the standard two-component formalism. We demonstrate that for this type of barrier a peculiar effect arises, i.e. the splitting of the electron wave function into two components inside the barrier, and we analyze its implications for the solution of the tunneling problem.

The paper is organized as follows. In Sec. II we briefly review the relevant features of the gauge model proposed in Refs. 39, 40, concentrating on the operators that account for the effects of the carbon background and the carbonfermion interaction. In this setting, we construct different types of barriers associated with defects in graphene, and discuss the implementation of the corresponding operators in the Dirac equation. Sec. III is dedicated to charge confinement. The transmission probability of an electron scattered off a barrier with sublattice symmetry breaking is investigated, and the conditions that enable the clustering of charge are identified. In Sec. IV, we focus our attention on the Klein tunneling, and consider the scattering off a barrier that describes a spatial distortion of the graphene sheet. As before, we discuss the angular behavior of the transmission coefficient, underlining the effects related to the mixing of the Dirac points. In Sec. V a short summary and the conclusions will be presented.

\section{THE THEORETICAL SETUP}

Let us begin by sketching the fermionic dynamics in graphene-based materials. Tight binding models provide a first approximation to describe the electronic properties of graphene, as they only take into account the fermionic degrees of freedom, whereas the carbon interactions are summarized via the hopping parameters which control the electron dynamics. Since the dynamical degrees of freedom in graphene must include both the fermions and carbon background, in ${ }^{39}$ a relativistic-like gauge model for graphene and nanotubes is suggested, which takes into account both the electron-hole and the carbon dynamics. In this framework, electrons are described by a four component Dirac-type spinor, while the carbon degrees of freedom are associated with a scalar field $\varphi$ and a gauge field $A^{\mu}$. Since the full description is somewhat long-winded, here we briefly present the main features of the model and direct the reader to $\stackrel{39}{9}$ for a full account. In this gauge model, the electron dynamics is described by the Dirac equation

$$
\left\{i \gamma^{\mu} D_{\mu}-P(\varphi)-P_{5}(\varphi) \gamma_{5}\right\} \psi=0
$$

where the polynomials $P(\varphi)$ and $P_{5}(\varphi)$ describe the interaction between fermions and the carbon crystal structure. Explicitly, the vacuum expectation value of the scalar field $\varphi$ vanishes for pure graphene, while for doped graphene $\langle\varphi\rangle=\varphi_{0} \neq 0$, where $\varphi_{0}$ is a minimum of the scalar potential $V\left(\varphi^{\dagger} \varphi\right)$. If one is able to fabricate graphene with islands where it is doped, one can simulated its electronic properties by taking

$$
P(\varphi)+P_{5}(\varphi) \gamma_{5}= \begin{cases}0 & \text { for undoped regions } \\ \left(g_{2}+i h_{2} \gamma_{5}\right) \varphi_{0}^{2} & \text { for doped regions }\end{cases}
$$

in the Dirac equation, where $g_{2}, h_{2}$ are the corresponding coupling constants. In this way, one reduces the problem to the investigation of the solutions of the Dirac equation in a square potential barrier, which includes the contribution Eq. (2.2) and can in principle account for any combination of Dirac $\gamma$ matrices. Furthermore, given that the potential barrier is associated with the doping of graphene, experiment can help to calibrate the numerical values of $g_{2} \varphi_{0}^{2}$ and $h_{2} \varphi_{0}^{2}$. The covariant derivative is $D_{\mu}=\partial_{\mu}+i g A_{\mu}$, and the four-component Dirac spinor is given by

$$
\psi=\left(\begin{array}{c}
\psi_{+}^{b} \\
\psi_{+}^{a} \\
\psi_{-}^{a} \\
\psi_{-}^{b}
\end{array}\right),
$$

where the upper index refers to the sublattices $A$ or $B$ and the lower index to the Dirac points $K$ (plus sign) and $K^{\prime}$ (minus sign). For the Dirac $\gamma$ matrices we will use the representation

$$
\gamma^{0}=\left(\begin{array}{cc}
0 & I \\
I & 0
\end{array}\right), \quad \gamma^{j}=\left(\begin{array}{cc}
0 & \sigma^{j} \\
-\sigma^{j} & 0
\end{array}\right), \quad \gamma_{5}=\left(\begin{array}{cc}
-I & 0 \\
0 & I
\end{array}\right),
$$




\begin{tabular}{ll}
\hline & Operators \\
\hline (S,S) & $I, \gamma_{0}, \gamma_{x} \gamma_{5}, \Sigma_{x}$ \\
(S,A) & $\gamma_{5}, \gamma_{x}, \sigma_{0 x}$ \\
$(\mathrm{~A}, \mathrm{~S})$ & $\gamma_{y}, \gamma_{z}, \sigma_{0 y}, \sigma_{0 z}$ \\
(A,A) & $\gamma_{y} \gamma_{5}, \gamma_{z} \gamma_{5}, \Sigma_{y}, \Sigma_{z}$ \\
\hline
\end{tabular}

TABLE I: The first column refers to the operator symmetry under interchange of sublattice and intervalley indices, respectively. The table assumes that the graphene sheet is on the $x-y$ plane. The $\Sigma$ matrices are defined as $\sigma^{i j}=-i \epsilon^{i j k} \Sigma_{k}$, where $\epsilon^{i j k}$ is the three dimensional Levi-Civita, $\epsilon^{123}=1$ and a sum over the index $k$ is implicit.

where $\sigma^{j}$ stands for the $j$ Pauli matrix. Since we are concerned with two dimensional fermions, the index $\mu$ takes the values $\mu=0,1,2$. The Dirac matrices satisfy $\left\{\gamma^{\mu}, \gamma^{\nu}\right\}=2 g^{\mu \nu}$.

In order to simulate other dynamical effects, which can be associated with the carbon structure or with the carbonelectron interaction, or to include the effects due to impurities/defects, new operators should be added to the Dirac equation (2.1). These operators can be, in principle, any of the remaining matrices $\gamma^{\mu}, \gamma^{\mu} \gamma_{5}, \sigma^{\mu \nu}$ (in this case the values of $\mu$ extend to the full four-dimensional Minkowski space). The symmetry properties of all the 16 operators under interchange of sublattices and of the Dirac points, respectively, are listed in Table 1 One can proceed and provide an interpretation for each of the 16 operators of the Dirac algebra in terms of graphene defects and corresponding barriers. In the following, we will not provide the detailed form of each contribution; instead, we will concentrate on the operators that have a simple physical interpretation, and represent the experimental situations considered in this work. If one adds a new term to the Dirac equation, let us say $-\Gamma \psi$, it contributes to the Hamiltonian of the system as $\bar{\psi} \Gamma \psi$, which can be rewritten in terms of the spinor components $\psi_{+}^{a}, \ldots$ For the particular choice of $\Gamma=\gamma^{z}$ (assuming that $x-y$ is the graphene plane) it follows that $\bar{\psi} \gamma^{z} \psi=\left[\left(\psi_{+}^{a}\right)^{\dagger} \psi_{+}^{a}+\left(\psi_{-}^{a}\right)^{\dagger} \psi_{-}^{a}\right]-\left[\left(\psi_{+}^{b}\right)^{\dagger} \psi_{+}^{b}+\left(\psi_{-}^{b}\right)^{\dagger} \psi_{-}^{b}\right]$. This term distinguishes the two carbon sublattices, it is antisymmetric under interchange of the sublattice indices, and favors the occupation of sublattice $B$ relative to sublattice $A$. Of course, if the coupling constant associated with this operator is negative, the occupation of sublattice $A$ is preferred. As discussed previously, the breaking of the sublattice symmetry can be realized by binding covalently foreign atoms with particular carbons in graphene and, in this way, a $\gamma^{z}$ operator can be simulated. It turns out that this operator (and its connection with 1D potential barriers) overcomes the Klein effect and leads to charge confinement. A second operator of interest for this work is the tensor interaction $\gamma^{1} \gamma^{2}=i \Sigma_{z}$, where $\Sigma_{z}$ is the third component of the spin operator (perpendicular on the plane of the graphene). It describes the rotation of the graphene around the $z$ axis and can be related to a topological defect of the lattice in graphene (see for example $\underline{\underline{4}}$ for a study of the low energy properties of graphene due to distortions of graphene sheets). Importantly, the aforementioned operators change the fermion dispersion relation, such that the electrons acquire an effective mass, with an energy gap twice the effective mass. For an explicit derivation of the fermion gap, and its correlation with the underlying scalar field, we refer the reader to Ref. 39 .

Since the coupling with the scalar field $\varphi$ devise the electronic properties of the doped regions of graphene, in the following we will set the gauge field $A_{\mu}=0$. After adding the relevant operators to the Dirac equation (2.1), we obtain:

$$
\left\{i \gamma^{\mu} \partial_{\mu}-D_{S}-i D_{P} \gamma^{5}-D_{0} \gamma^{0}-D_{V} \gamma^{z}-i D_{T} \gamma^{1} \gamma^{2}\right\} \psi=0,
$$

where we have introduced the notations $D_{S}=P(\varphi)$ and $D_{P}=-i P_{5}(\varphi)$ for the scalar and pseudoscalar interactions, respectively. $D_{T(V)}$ denotes the strength of the tensor (vector) interaction, and the term proportional to $\gamma_{0}$ can be viewed as a chemical potential. Starting with the above equation, the task is now to investigate the transmission of the electrons through a 1D barrier, and establish under which conditions the charge confinement and Klein tunneling take place.

\section{CHARGE CONFINEMENT}

In this section we restrict the analysis to a set of operators that include the scalar, pseudoscalar and vector barriers in the Dirac equation. Setting $D_{T}=0$, our starting Dirac equation takes the form

$$
\left\{i \gamma^{\mu} \partial_{\mu}-D_{S}-i D_{P} \gamma^{5}-D_{0} \gamma^{0}-D_{V} \gamma^{z}\right\} \psi=0
$$

The scattering over an electrostatic barrier (within the two-component formalism) was already investigated in Ref. 6 for single layer and double layer graphene, and the Klein paradox has been demonstrated. When one takes into account in the Dirac equation the pseudoscalar interaction $\bar{\psi} \gamma_{5} \psi$, the sublattice symmetry breaking operator $\bar{\psi} \gamma^{z} \psi$ 


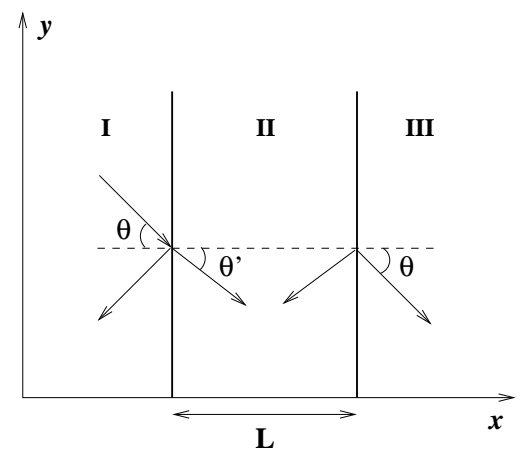

FIG. 1: Sketch of a one-dimensional defect in an infinite slab of graphene. $\theta$ and $\theta^{\prime}$ are the angles used in the scattering problem in regions $I$ (pure graphene), II (graphene with defect), III (pure graphene), and $L$ is the width of the barrier.

and the scalar term $\bar{\psi} \psi$, the scattering pattern computed in Ref. 6 changes dramatically. In particular, the tunneling associated with the Klein effect can be avoided, leading to charge confinement.

Following $\frac{6}{6}$, we will consider the simple geometry of an 1D defect in graphene, where the doping occurs only for the region for $0<x<L$. The "experimental" setup is illustrated in Fig. 1. The wave function describing an incident electron with energy $E$ and momentum $\vec{p}=E\left(\cos \theta \hat{e}_{x}+\sin \theta \hat{e}_{y}\right)$, in the region $I(x<0)$, is given by

$$
\psi_{I}(x)=e^{i \vec{p} \cdot \vec{x}}\left(\begin{array}{c}
A_{K} \\
-A_{K} e^{i \theta} \\
B_{K^{\prime}} e^{-i \theta} \\
B_{K^{\prime}}
\end{array}\right)+e^{i \vec{p}^{\prime} \cdot \vec{x}}\left(\begin{array}{c}
R_{K} \\
R_{K} e^{-i \theta} \\
-R_{K^{\prime}} e^{i \theta} \\
R_{K^{\prime}}
\end{array}\right),
$$

where the momentum $\vec{p}^{\prime}=E\left(-\cos \theta \hat{e}_{x}+\sin \theta \hat{e}_{y}\right)$ corresponds to the solution associated with the reflected wave in region $I$. For $0<x<L$ one has

$$
\psi_{I I}(x)=e^{i \vec{q} \cdot \vec{x}}\left\{a_{K}\left(\begin{array}{c}
1 \\
0 \\
\mathcal{D}_{+} \\
\mathcal{D}_{q} e^{i \theta^{\prime}}
\end{array}\right)+b_{K^{\prime}}\left(\begin{array}{c}
0 \\
1 \\
\mathcal{D}_{q} e^{-i \theta^{\prime}} \\
\mathcal{D}_{-}
\end{array}\right)\right\}+e^{i \vec{q}^{\prime} \cdot \vec{x}}\left\{c_{K}\left(\begin{array}{c}
1 \\
0 \\
\mathcal{D}_{+} \\
-\mathcal{D}_{q} e^{-i \theta^{\prime}}
\end{array}\right)+d_{K^{\prime}}\left(\begin{array}{c}
0 \\
1 \\
-\mathcal{D}_{q} e^{i \theta^{\prime}} \\
\mathcal{D}_{-}
\end{array}\right)\right\},
$$

with

$$
\mathcal{D}_{ \pm}=\frac{E-D_{0} \pm D_{V}}{D_{S}+i D_{P}} \quad \text { and } \quad \mathcal{D}_{q}=\frac{|\vec{q}|}{D_{S}+i D_{P}} .
$$

As before, $\vec{q}=|\vec{q}|\left(\cos \theta^{\prime} \hat{e}_{x}+\sin \theta^{\prime} \hat{e}_{y}\right)$ and $\vec{q}^{\prime}=|\vec{q}|\left(-\cos \theta^{\prime} \hat{e}_{x}+\sin \theta^{\prime} \hat{e}_{y}\right)$. The Dirac equation has a nontrivial solution in region $I I$ if the corresponding determinant vanishes, which leads to the condition

$$
\vec{q}^{2}=\left(E-D_{0}\right)^{2}-D_{V}^{2}-D_{S}^{2}-D_{P}^{2}
$$

As shall shortly become clear, it is important to notice that the component $q_{x}=\sqrt{\vec{q}^{2}-E^{2} \sin ^{2} \theta}$ can be real or a pure imaginary number, depending on the parameters entering Eq. 3.5. and the angle of incidence. For a real $q_{x}$ the Klein tunneling is allowed and the barrier is perfectly transparent for certain angles of incidence. On the other hand, when $q_{x}$ is imaginary the barrier becomes opaque and transmission is highly suppressed. For $x>L$ the wave function is given by

$$
\psi_{I I I}(x)=e^{i \vec{p} \cdot \vec{x}}\left(\begin{array}{c}
t_{K} \\
-t_{K} e^{i \theta} \\
t_{K^{\prime}} e^{-i \theta} \\
t_{K^{\prime}}
\end{array}\right)
$$

For a relativistic system the wave function is continuous everywhere, therefore $\psi_{I}(x=0)=\psi_{I I}(x=0)$ and $\psi_{I I}(x=L)=\psi_{I I I}(x=L)$. From the matching conditions and with the definition of the transmission probability

$$
T(\theta)=\frac{\left|t_{K}\right|^{2}+\left|t_{K^{\prime}}\right|^{2}}{\left|A_{K}\right|^{2}+\left|B_{K^{\prime}}\right|^{2}},
$$



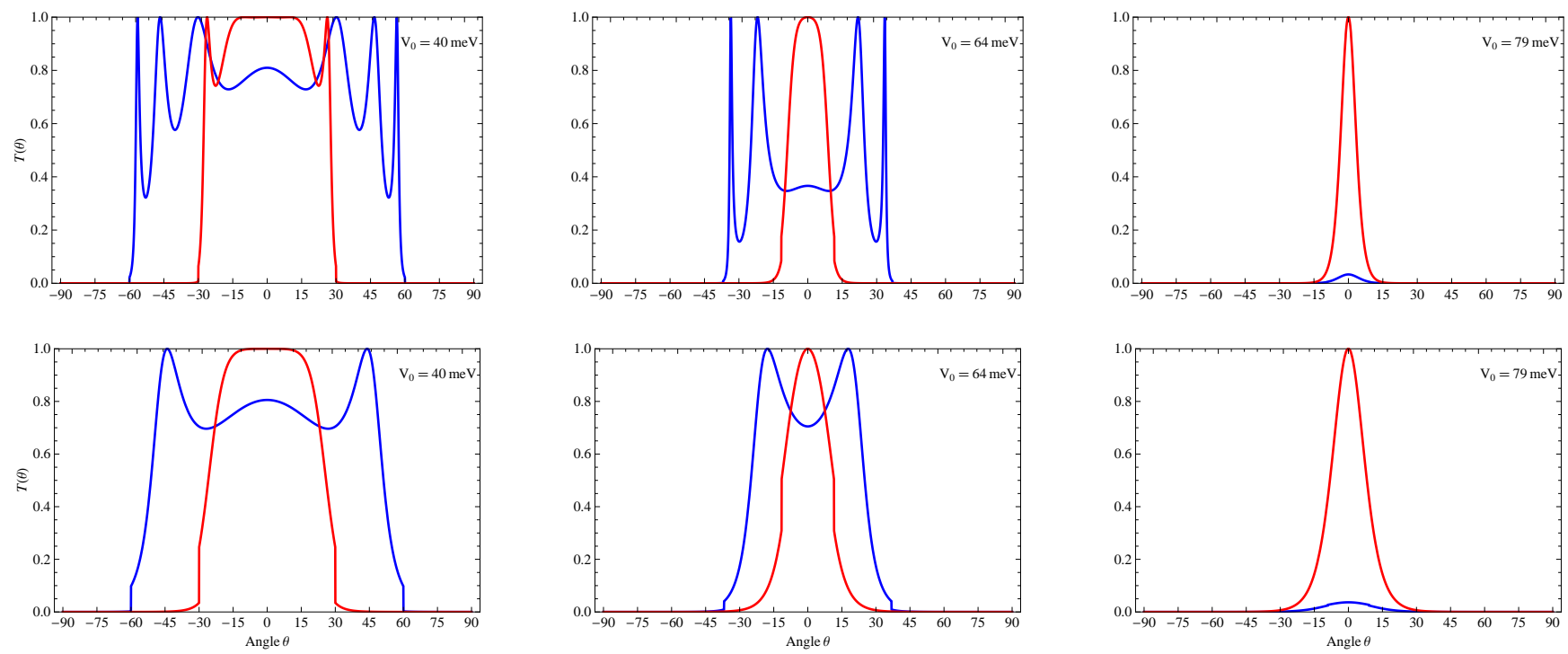

FIG. 2: (Color online) Angular behavior of the transmission probability $T(\theta)$ for a $\gamma^{z}$ barrier (blue line) plotted against a $\gamma^{0}$ barrier (red line), for the incoming energy $E=80 \mathrm{meV}$ and different heights of the barrier. The widths of the barriers are $L=110 \mathrm{~nm}$ (upper panel) and $L=50 \mathrm{~nm}$ (lower panel). In the figures, $V_{0}$ denotes the height of the barriers, i.e. $V_{0}=D_{0}$ for the $\gamma^{0}$ barrier and $V_{0}=D_{V}$ for the $\gamma^{z}$ barrier (in general, in the second case $V_{0}=\sqrt{D_{S}^{2}+D_{P}^{2}+D_{V}^{2}}$ if one takes into account all barriers associated with graphene doping; see also text for details). The $\gamma^{0}$ barriers reproduce the results found in the two-component formalism, as described in Refs. 6. 10.

one obtains the following expression:

$$
T(\theta)=\frac{\vec{q}^{2} \cos ^{2} \theta \cos ^{2} \theta^{\prime}}{\vec{q}^{2} \cos ^{2}\left(q_{x} L\right) \cos ^{2} \theta \cos ^{2} \theta^{\prime}+\sin ^{2}\left(q_{x} L\right)\left(E-D_{0}-|\vec{q}| \sin \theta \sin \theta^{\prime}\right)^{2}},
$$

where $\theta^{\prime}=\arctan \left(p_{y} / q_{x}\right)$. In the above formula, we notice that the the Dirac points $K$ and $K^{\prime}$ do not mix, in the sense that terms of the type $A_{K} B_{K^{\prime}}^{*}$ are not present. Further, note that the transmission probability corresponding to the $\gamma^{0}$ operator, with $D_{S}=D_{P}=D_{V}=0$, reproduces the solution obtained in Ref. 6 within the two-component formalism (stemming from the four-component formalism, when the couplings between components are discarded). We remind the reader that in this case the result (3.8) reduces to:

$$
T_{0}(\theta)=\frac{\cos ^{2} \theta \cos ^{2} \theta^{\prime}}{\cos ^{2}\left(q_{x} L\right) \cos ^{2} \theta \cos ^{2} \theta^{\prime}+\sin ^{2}\left(q_{x} L\right)\left(1-s \sin \theta \sin \theta^{\prime}\right)^{2}},
$$

where $s=\operatorname{sgn}\left(E-D_{0}\right)$.

Since the $\gamma_{0}$ interaction is not relevant for our purpose to demonstrate charge confinement, in the following we will set $D_{0}=0$. The potentials $D_{S}, D_{P}$ and $D_{V}$ all generate an electronic massive-type dispersion relation, see Eq. (3.5). In this sense, the doping associated with any of the scalar, pseudoscalar and $\gamma^{z}$ operators change the inertia of the charge carriers in a similar way. More, given that the $I, \gamma_{5}$ and $\gamma^{z}$ contributions in Eq. (3.5) are symmetric, it is enough to study one of the three barriers, say that associated with the $\gamma^{z}$ interaction $D_{V}$ (which, as discussed, breaks the sublattice symmetry), while setting $D_{S}$ and $D_{P}$ to zero. Then the condition Eq. (3.5) simplifies to

$$
\vec{q}^{2}=E^{2}-D_{V}^{2}
$$

The apparent singularities in the wave function, Eq. (3.3), can be discarded simply by multiplying the spinor with a factor $\left(D_{S}+i D_{P}\right)$, which changes its length but does not affect the physical results. Moreover, we have checked that setting $D_{S}=D_{P}=D_{V}=0$ directly in the Dirac equation leads to the transmission coefficient $T_{0}(\theta)$, Eq. (3.9), although the solutions of the Dirac equation inside the barrier look rather different.

In Fig. 2 the angular behavior of the transmission probability for a $\gamma^{z}$ barrier, Eq. (3.8), with $\vec{q}^{2}$ given by Eq. (3.10), (blue line) is plotted against the $\gamma^{0}$ barrier, Eq. (3.9) (red line), for various heights and for two barrier lengths. As shown in the left panels of Fig. 2 for a $\gamma^{z}$ barrier lower than the energy of the incident electron, there are several directions for which the barrier is transparent. This is the "standard" Klein tunneling - already demonstrated for a 
$\gamma_{0}$ barrier $\frac{6,10}{2}$ - which occurs due to the fact that the wave function inside the barrier, Eq. (3.3), has an oscillating behavior (the momentum $|\vec{q}|$ is real in this case), just like the wave function outside the barrier. Considering normal incidence $\left(\theta=\theta^{\prime}=0\right)$ and keeping $D_{V}<E$, we notice that the $\gamma^{z}$ barrier is not perfectly transparent, as opposed to the $\gamma^{0}$ barrier. Instead, transparency occurs only for higher angles of incidence. On a $\gamma^{0}$ barrier, for $\theta=\theta^{\prime}=0$, $T_{0}(0)=1$ for any value of $q_{x} L$, independent of the height of the barrier, as can be seen from Fig. 2 and from Eq. (3.9), whereas in a $\gamma^{z}$ barrier, $T(0)$ is no longer unit but depends on the ratio $E / D_{V}$. A straightforward calculation gives

$$
T(0)=\frac{1}{1+\frac{D_{V}^{2}}{E^{2}-D_{V}^{2}} \sin ^{2}\left(q_{x} L\right)}
$$

and therefore $T(0)<1$, for $D_{V}<E$. Increasing the barrier height, the range of angles where the perfect tunneling through a $\gamma^{z}$ barrier is allowed shrinks until the Klein tunneling is suppressed, as can be seen in the last plots of Fig. 2. This behavior can be understood by looking at Eq. (3.8). Indeed, setting $E=D_{V}$, it follows that $\vec{q}^{2}=0$ and $T(\theta)=0$ for any angle of incidence. When the height of the barrier is higher then the electron energy, the momentum $\vec{q}^{2}$, Eq. (3.10), flips the sign such that $\sqrt{\vec{q}^{2}}$ and the component $q_{x}$ become imaginary, and the wave function inside the barrier switches from oscillating to exponential decay. In turn, this implies that for barriers higher than the electron energy, the barrier becomes opaque, thus leading to the charge clustering of the electrons.

\section{KLEIN TUNNELING AND INTERVALLEY MIXING}

So far, in the scattering off a barrier of scalar, pseudoscalar and vector type, the transitions $K \leftrightarrow K^{\prime}$ did not occur, in the sense that a term that mixes the coefficients corresponding to the two different valleys was not present in the transition amplitude. In the present section, we investigate the effects on Klein tunneling emerging from the $K \leftrightarrow K^{\prime}$ transitions, by introducing a tensor term proportional to $\gamma^{1} \gamma^{2}=i \Sigma_{z}$ (and discard all other contributions), where $\Sigma_{z}$ is the third component of the spin operator in the four-spinor representation. Recall that such an operator can be associated with a rotation of the honeycomb carbon lattice. In this case, the Dirac equation (2.5) takes the form

$$
\left\{i \gamma^{\mu} \partial_{\mu}-i D_{T} \gamma^{1} \gamma^{2}\right\} \psi=0
$$

As before we will consider the geometry of an almost $1 \mathrm{D}$ defect in graphene, such that the electron is scattered off a barrier that ranges between 0 and $L$ (see also Fig. (1). For an incident electron moving with energy $E$ and momentum $\vec{p}=E\left(\cos \theta \hat{e}_{x}+\sin \theta \hat{e}_{y}\right)$, the wave functions in the regions $I(x<0)$ and $I I I(x>L)$ are given by Eqs. (3.2) and (3.6). Inside the barrier, where the contribution of the tensor interaction is non-vanishing, a plane wave nontrivial solution for the Dirac equation requires

$$
D_{T}^{2}=(E \pm|\vec{q}|)^{2} .
$$

For a given electron energy $E>0$ there are two possible momenta, $\left|\vec{q}^{(1,2)}\right|=\left|E \pm D_{T}\right|$, with the associated spinors given by, respectively,

$$
\psi^{(1)}=\left(\begin{array}{c}
1 \\
-e^{i \theta^{(1)}} \\
-1 \\
-e^{i \theta^{(1)}}
\end{array}\right), \quad \psi^{(2)}=\left(\begin{array}{c}
1 \\
-e^{i \theta^{(2)}} \\
1 \\
e^{i \theta^{(2)}}
\end{array}\right)
$$

This means that, in the scattering through the 1D tensor barrier, the incoming wave is divided inside the region II, see Fig. 1 into two distinct plane waves, propagating with different momenta and at different angles, $\theta^{(1)}$ and $\theta^{(2)}$. Since one wave is exponentially enhanced and the other, exponentially suppressed, their mixing will produce a pattern that exhibits perfect tunneling for several angles, regardless of the height of the barrier. This is in contrast to the $\gamma_{z}$ type of defect considered in the previous section, where for a barrier higher than the electron energy the transmission probability goes very rapidly to zero. Using Eq. (4.3), we can write down the general solution of the Dirac equation for $0<x<L$ :

$$
\psi_{I I}(x)=e^{i \vec{q}^{(1)} \cdot \vec{x}} \alpha_{K}\left(\begin{array}{c}
1 \\
-e^{i \theta^{(1)}} \\
-1 \\
-e^{i \theta^{(1)}}
\end{array}\right)+e^{i \vec{q}^{(2)} \cdot \vec{x}} \beta_{K^{\prime}}\left(\begin{array}{c}
1 \\
-e^{i \theta^{(2)}} \\
1 \\
e^{i \theta^{(2)}}
\end{array}\right)+e^{i \vec{q}^{(1)} \cdot \vec{x}} \eta_{K}\left(\begin{array}{c}
1 \\
e^{-i \theta^{(1)}} \\
-1 \\
e^{-i \theta^{(1)}}
\end{array}\right)+e^{i \vec{q}^{(2)} \cdot \vec{x}} \lambda_{K^{\prime}}\left(\begin{array}{c}
1 \\
e^{-i \theta^{(2)}} \\
1 \\
-e^{-i \theta^{(2)}}
\end{array}\right),
$$


where $\vec{q}^{(1,2)}=\left|\vec{q}^{(1,2)}\right| e^{i \theta^{(1,2)}}$ and $\vec{q}^{\prime(1,2)}=-\left|\vec{q}^{(1,2)}\right| e^{-i \theta^{(1,2)}}$.

As in the previous section, we use the fact that a solution of the Dirac equation is a continuous function everywhere, in order to obtain the boundary conditions for the wave function $\psi$. For the tensor barrier, it is convenient to write the boundary conditions at the borders of region $I I$ in matrix form. At $x=0$ we have

$$
\left(\begin{array}{cccc}
1 & 0 & 1 & 0 \\
-e^{i \theta} & 0 & e^{-i \theta} & 0 \\
0 & 1 & 0 & 1 \\
0 & e^{i \theta} & 0 & -e^{-i \theta}
\end{array}\right)\left(\begin{array}{c}
A_{K} \\
B_{K^{\prime}} \\
R_{K} \\
R_{K^{\prime}}
\end{array}\right)=\left(\begin{array}{cccc}
1 & 1 & 1 & 1 \\
-e^{i \theta^{(1)}} & -e^{i \theta^{(2)}} & e^{-i \theta^{(1)}} & e^{-i \theta^{(2)}} \\
-1 & 1 & -1 & 1 \\
-e^{i \theta^{(1)}} & e^{i \theta^{(2)}} & e^{-i \theta^{(1)}} & -e^{-i \theta^{(2)}}
\end{array}\right)\left(\begin{array}{c}
\alpha_{K} \\
\beta_{K^{\prime}} \\
\eta_{K} \\
\lambda_{K^{\prime}}
\end{array}\right) .
$$

With the notations

$$
M=\left(\begin{array}{cccc}
1 & 0 & 1 & 0 \\
-e^{i \theta} & 0 & e^{-i \theta} & 0 \\
0 & 1 & 0 & 1 \\
0 & e^{i \theta} & 0 & -e^{-i \theta}
\end{array}\right) \text { and } N=\left(\begin{array}{cccc}
1 & 1 & 1 & 1 \\
-e^{i \theta^{(1)}} & -e^{i \theta^{(2)}} & e^{-i \theta^{(1)}} & e^{-i \theta^{(2)}} \\
-1 & 1 & -1 & 1 \\
-e^{i \theta^{(1)}} & e^{i \theta^{(2)}} & e^{-i \theta^{(1)}} & -e^{-i \theta^{(2)}}
\end{array}\right)
$$

we can rewrite the above equation as

$$
M\left(\begin{array}{c}
A_{K} \\
B_{K^{\prime}} \\
R_{K} \\
R_{K^{\prime}}
\end{array}\right)=N\left(\begin{array}{c}
\alpha_{K} \\
\beta_{K^{\prime}} \\
\eta_{K} \\
\lambda_{K^{\prime}}
\end{array}\right)
$$

Similarly, the boundary condition in $x=L$ reads:

$$
e^{i p_{x} L} M\left(\begin{array}{c}
t_{K} \\
t_{K^{\prime}} \\
r_{K} \\
r_{K^{\prime}}
\end{array}\right)=N\left(\begin{array}{cccc}
e^{i q_{x}^{(1)} L} & 0 & 0 & 0 \\
0 & e^{i q_{x}^{(2)} L} & 0 & 0 \\
0 & 0 & e^{-i q_{x}^{(1)} L} & 0 \\
0 & 0 & 0 & e^{-i q_{x}^{(2)} L}
\end{array}\right)\left(\begin{array}{c}
\alpha_{K} \\
\beta_{K^{\prime}} \\
\eta_{K} \\
\lambda_{K^{\prime}}
\end{array}\right)
$$

Notice the insertion of the coefficients $r_{K}, r_{K^{\prime}}$ in the transmitted wave. Even though this alteration does not change the final result, there are two reasons that motivate it. Firstly, with this modification the matrix that multiplies the outgoing spinor becomes identical to the matrix $M$ in Eq. (4.7), corresponding to the incoming wave - otherwise, if $r_{K}=r_{K^{\prime}}=0$ the matrix $M$ should be modified such that the last two columns are zero, although formally they can be left unchanged since the multiplication with zero does not change the final result. A second (physical) reason is related to the possibility of investigating a double (or multiple) square potential, where the wave emerging from the first barrier does have a reflected component, which is then scattered on a second barrier, and so forth. With the notation

$$
\tilde{L}=\left(\begin{array}{cccc}
e^{i q_{x}^{(1)} L} & 0 & 0 & 0 \\
0 & e^{i q_{x}^{(2)} L} & 0 & 0 \\
0 & 0 & e^{-i q_{x}^{(1)} L} & 0 \\
0 & 0 & 0 & e^{-i q_{x}^{(2)} L}
\end{array}\right)
$$

and putting Eqs. (4.7), (4.8) together, we find that the relation between the coefficients corresponding to the incoming and outgoing waves can be written in matrix form as (here we set $r_{K}, r_{K^{\prime}}$ back to zero and remove the phase factor $e^{i p_{x} L}$, which does not contribute to the transition amplitude)

$$
\left(\begin{array}{c}
t_{K} \\
t_{K^{\prime}} \\
0 \\
0
\end{array}\right)=M^{-1} N \tilde{L} N^{-1} M\left(\begin{array}{c}
A_{K} \\
B_{K^{\prime}} \\
R_{K} \\
R_{K^{\prime}}
\end{array}\right) .
$$

Furthermore, in order to calculate the transmission probability, one can rewrite the above equation in a form that eliminates the dependence on the reflection coefficients $R_{K}, R_{K^{\prime}}$. With the notation

$$
X=M^{-1} N \tilde{L} N^{-1} M
$$



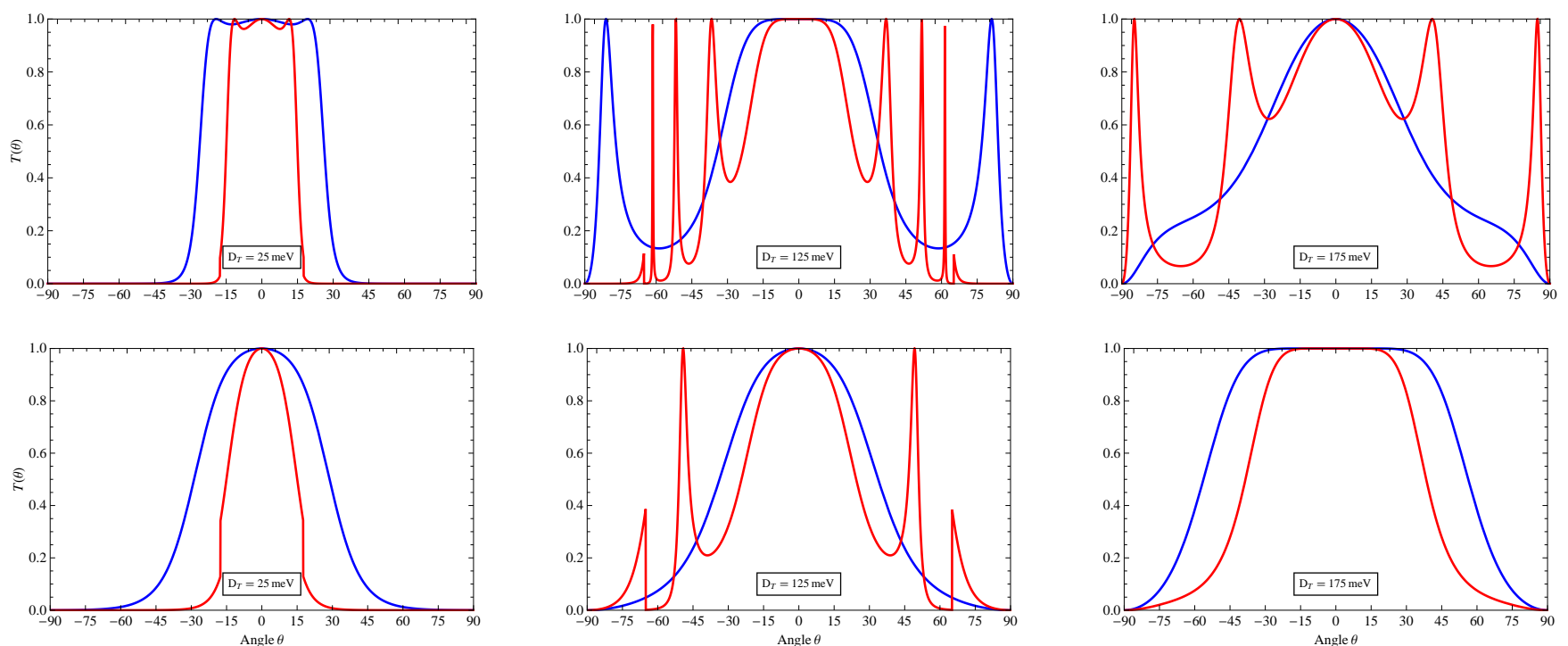

FIG. 3: (Color online) Angular behavior of the transmission probability $T(\theta)$ for a tensor barrier $\gamma^{1} \gamma^{2}=i \Sigma_{z}$ (blue line) plotted against a $\gamma^{0}$ barrier (red line), for the incoming energy $E=50 \mathrm{meV}$ and different heights of the barrier. The widths of the barrier are $L=110 \mathrm{~nm}$ (upper panel) and $L=50 \mathrm{~nm}$ (lower panel), and $D_{T}$ denotes the height of the barrier.

one can split the equation (4.10) into "blocks", and rewrite it in the following form:

$$
\begin{aligned}
\left(\begin{array}{c}
t_{K} \\
t_{K^{\prime}}
\end{array}\right) & =X_{11}\left(\begin{array}{c}
A_{K} \\
B_{K^{\prime}}
\end{array}\right)+X_{12}\left(\begin{array}{c}
R_{K} \\
R_{K^{\prime}}
\end{array}\right), \\
\left(\begin{array}{c}
0 \\
0
\end{array}\right) & =X_{21}\left(\begin{array}{c}
A_{K} \\
B_{K^{\prime}}
\end{array}\right)+X_{22}\left(\begin{array}{c}
R_{K} \\
R_{K^{\prime}}
\end{array}\right),
\end{aligned}
$$

where $X_{i j}$ are $2 \times 2$ matrices, corresponding to the four entries of the matrix $X$, Eq. (4.11). It is straightforward to show that

$$
\left(\begin{array}{c}
t_{K} \\
t_{K^{\prime}}
\end{array}\right)=\left(X_{11}-X_{12} X_{22}^{-1} X_{21}\right)\left(\begin{array}{c}
A_{K} \\
B_{K^{\prime}}
\end{array}\right)
$$

and hence we have an explicit relation between the coefficients that contribute to the transmission probability. We have used Mathematica to tackle our problem and the calculation shows a matrix $X_{11}$ with non-vanishing off diagonal terms, i.e. that produce a mixing between the coefficients $A_{K}$ and $B_{K^{\prime}}$. This implies that a rotation of the honeycomb carbon lattice induces an intervalley transition $\left(K \leftrightarrow K^{\prime}\right)$ in the barrier reflection and transmission. The various momenta and angles entering the above equations are given by

$$
\begin{aligned}
& p_{x}=E \cos \theta, p_{y}=E \sin \theta, \\
& q_{x}^{(1,2)}=\left|\vec{q}^{(1,2)}\right| \cos \theta^{(1,2)}, q_{y}^{(1)}=q_{y}^{(2)}=p_{y}=\left|\vec{q}^{(1)}\right| \sin \theta^{(1)}=\left|\vec{q}^{(2)}\right| \sin \theta^{(2)}, \\
& \theta^{(1,2)}=\arcsin \frac{E \sin \theta}{E \pm D_{T}},
\end{aligned}
$$

such that the only free parameters are the incidence angle $\theta$, the width of the barrier $L$ and the strength of the interaction $D_{T}$. The transmission coefficient is then calculated numerically, using the definition Eq. (3.7) and the relation Eq. (4.14).

In order to investigate the Klein tunneling, including the effects of the $K \leftrightarrow K^{\prime}$ mixing, in Figs. 35 we plot the transmission probability for a tensor barrier (blue line) against a $\gamma^{0}$ barrier given by Eq. (3.9) (red line). For a lower value of the electron energy $(E=50 \mathrm{meV})$, we notice that for a relatively low barrier (half of the electron energy), the tensor barrier has a higher acceptance than the $\gamma^{0}$ barrier - this is mostly visible for a width of $L=110 \mathrm{~nm}$, where the transmission coefficient is close to one for $\theta \leq 38^{\circ}$ for tensor and $\theta \leq 17^{\circ}$ for $\gamma^{0}$ barrier, as can be seen in Fig. 3. Increasing the height, the $\gamma^{0}$ barrier picks up more "structure", becoming transparent for central incidence and at several special angles, whereas the tensor interaction shows a less rich "structure". For even higher barrier the behavior follows the same trend except that there is a smaller number of angles for which perfect tunneling is 

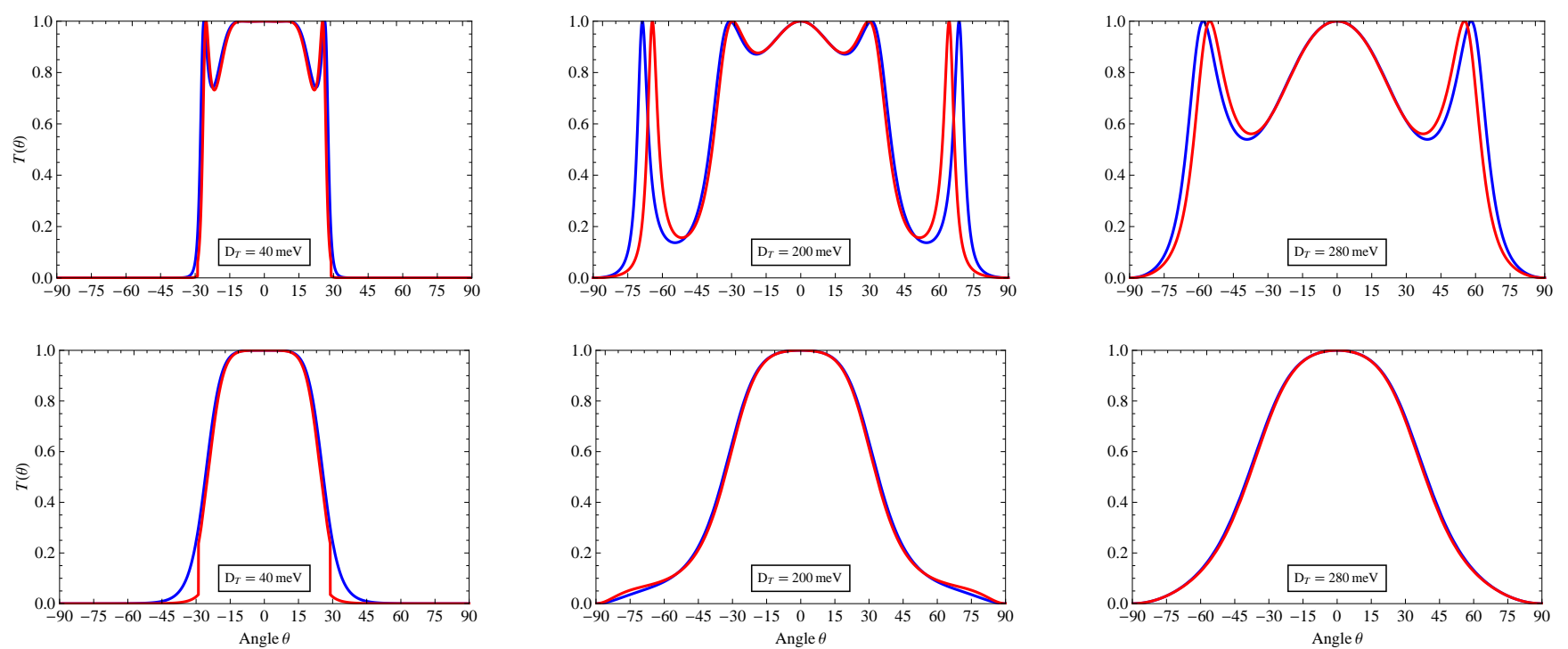

FIG. 4: (Color online) Same as in Fig. 3 for an energy of the incoming electron $E=80$ meV. The widths of the barrier are $L=110 \mathrm{~nm}$ (upper panel) and $L=50 \mathrm{~nm}$ (lower panel), and $D_{T}$ denotes the height of the barrier.
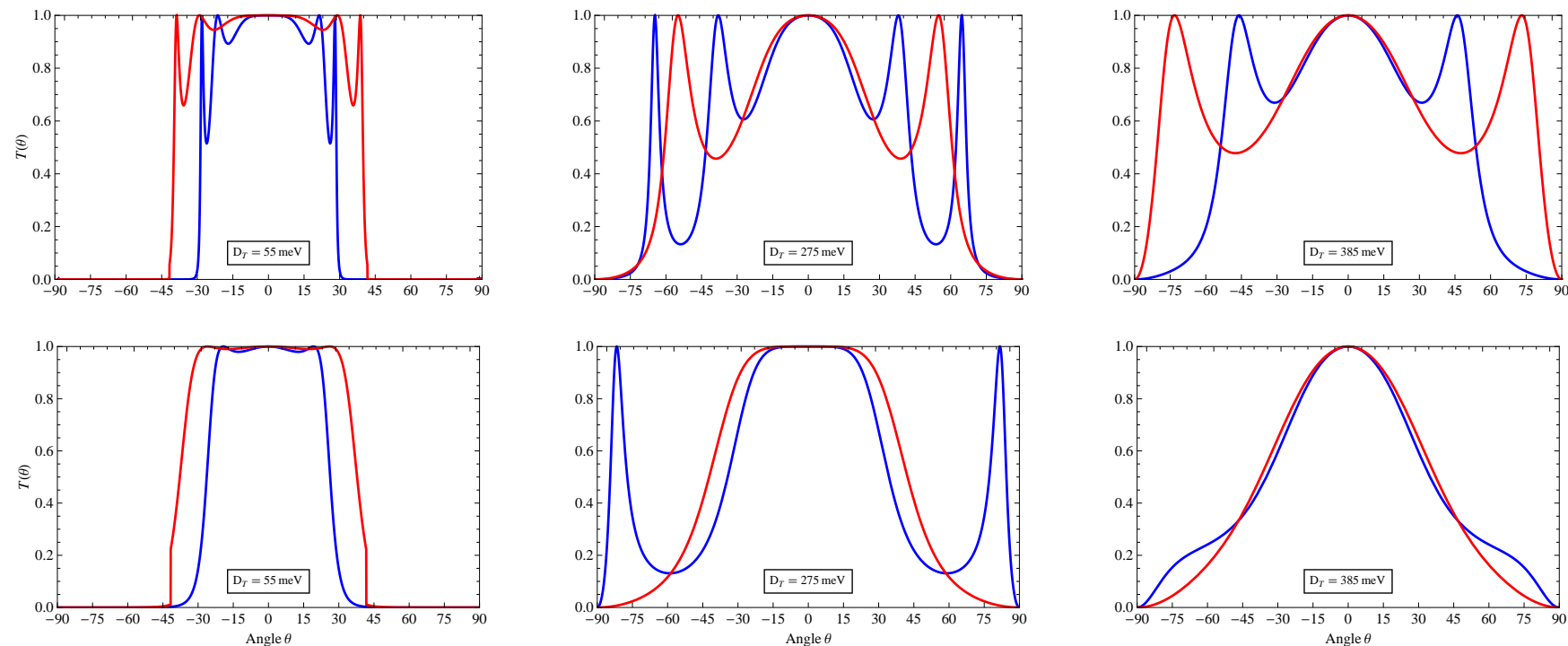

FIG. 5: (Color online) Same as in Fig. 3 for an energy of the incoming electron $E=110$ meV. The widths of the barrier are $L=110 \mathrm{~nm}$ (upper panel) and $L=50 \mathrm{~nm}$ (lower panel), and $D_{T}$ denotes the height of the barrier.

allowed. In the second set of plots (depicted in Fig. (4) we increase the electron energy, while keeping the widths of the barriers constant. Choosing the electron energy identical to the value used in the calculation presented in $\underline{10}(E=80$ $\mathrm{meV}$ ), we find that the transmission probabilities for tensor and $\gamma^{0}$ barriers are almost identical. Finally, inspecting

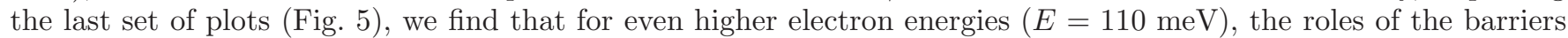
are reversed: for a lower height, the tensor barrier has a lower acceptance compared with the $\gamma^{0}$ barrier, whereas for higher barriers the tensor component exhibits more angles for which perfect tunneling takes place. Our results show that the effects of the $K \leftrightarrow K^{\prime}$ mixing, combined with the splitting of the wave inside the barrier, are more prominent for higher energies of the incoming electron. We speculate that this is a result of the fact that the exponential factors of the wave function inside the barrier, Eq. (4.4), depend on the absolute value of the electron energy $E$ (and the barrier height $D_{T}$ ), whereas the angles Eq. 4.17) only depend on the ration $E / D_{T}$. 


\section{SUMMARY AND CONCLUSIONS}

In this paper, we have used the four component spinor formalism to investigate the transmission coefficients for $\gamma^{z}$, $\gamma^{0}, I, \gamma_{5}$ and $\gamma^{1} \gamma^{2}$ one-dimensional barriers in graphene. We have worked in the framework of the gauge theoretical model proposed in Refs. 39,40, where the electron-hole and carbon dynamics are simulated by a scalar and gauge fields. The different operators in the Dirac equation can be related to various types of defects in graphene: the $\gamma^{z}$ operator is related to the breaking of the sublattice symmetry, $\gamma^{1} \gamma^{2}$ describes a topological defect of the graphene sheet, whereas $\gamma^{0}$ can be associated with a chemical potential, and $I$ and $\gamma_{5}$ appear in effective gauge theories for graphene 39.40 .

For the $\gamma^{0}$ barrier we reproduce the results of the previous calculations performed within the two component spinor formalism ${ }^{6}$. For the $\gamma^{z}, I$ and $\gamma_{5}$ barriers we found that the transmittance has a strong dependence on the barrier height and incidence angle of the electron. In particular, under certain conditions these interactions generate an opaque barrier which gives rise to charge confinement. From an experimental point of view, the most prominent example is the breaking of the sublattice symmetry associated with the $\gamma^{z}$ operator, which can be implemented via covalent bonding of foreign atoms to carbon atoms. Hence, according to our calculation, chemical bonding can be used as an instrument for controlling charge clustering in graphene. The remaining one-dimensional barrier, corresponding to the tensor interaction $\gamma^{1} \gamma^{2}=i \Sigma_{3}$, exhibits an unusual feature. Specifically, it appears that the wave function of the incoming electron is divided into two plane waves inside the barrier, propagating at different angles and with different momenta. This translates into a distinct behavior of the transmission probability as a function of the incidence angle of the electron: while the Klein tunneling is observed just like in the case of the $\gamma^{0}$ barrier $\underline{6.10}$, for particular values of the energy the scattering patterns of the two barriers can differ significantly (see also Figs. 3 50). We hope that understanding the nature of the tunneling states will be of further use in studies of the transport properties of graphene-based devices.

\section{ACKNOWLEDGMENTS}

The authors acknowledge financial support from the Brazilian agencies FAPESP (Fundação de Amparo à Pesquisa do Estado de São Paulo), CNPq (Conselho Nacional de Desenvolvimento Científico e Tecnológico). OO and CP acknowledge financial support from the Portuguese agency FCT (Fundação para a Ciência e Tecnologia) under contract PTDC/FIS/100968/2008. CP also acknowledges support from the Deutsche Forschungsgemeinschaft through contract SFB 634 .

${ }^{1}$ K. S. Novoselov, A. K. Geim, S. V. Morozov, D. Jiang, Y. Zhang, S. V. Dubonos, I. V. Grigorieva, and A. A. Firsov, Science 306, 666 (2004).

2 K. S. Novoselov, A. K. Geim, S. V. Morozov, D. Jiang, M. I. Katsnelson, I. V. Grigorieva, S. V. Dubonos, and A. A. Firsov, Nature (London) , 438, 197 (2005).

3 V. P. Gusynin, and S. G. Sharapov, Physical Review Letters, 95, 146801 (2005).

4 Y. Zhang, Y.-W. Tan, H. L. Stormer, and P. Kim, Nature 438, 201 (2005).

${ }^{5}$ V. V. Cheianov, and V. I. Fal'Ko, Phys. Rev. B 74, 041403 (2006).

${ }_{6}$ M. I. Katsnelson, K. S. Novoselov, and A. K. Geim, Nature Physics 2, 620 (2006).

7 A. V. Rozhkov, G. Giavaras, Y. P. Bliokh, V. Freilikher, and F. Nori, Phys. Rep. 503, 77 (2011).

8 P. R. Wallace, Phys. Rev. 71, 622 (1947).

${ }^{9}$ C. Berger, Z. Song, X. Li, X. Wu, N. Brown, C. Naud, D. Mayou, T. Li, J. Hass, A. N. Marchenkov, E. H. Conrad, P. N. First, and W. A. de Heer, Science 312, 1191 (2006).

10 A. Castro Neto, F. Guinea, N. Peres, K. Novoselov, and A. Geim, Rev. Mod. Phys. 81, 109 (2009).

11 N. M. R. Peres, Rev. Mod. Phys. 82, 2673 (2010).

12 D. S. L. Abergel, V. Apalkov, J. Berashevich, K. Ziegler, and T. Chakraborty, Adv. Phys. 59, 261 (2010).

${ }^{13}$ V. N. Kotov, B. Uchoa, V. M. Pereira, A. H. Castro Neto, F. Guinea, arXiv:1012.3484 [cond-mat].

14 S. Das Sarma, S. Adam, E. H. Hwang, E. Rossi, Rev. Mod. Phys. 83, 407 (2011).

15 F. Banhart, J. Kotakoski, and A. V. Krasheninnikov, ACS Nano 5, 26 (2011).

16 J. Lahiri, Y. Lin, P. Bozkurt, I. I. Oleynik and M. Batzill, Nature Nano 53, 326 (2010).

17 O. Klein, Z. Phys. 53, 157 (1929).

18 R. K. Su, G. G. Siu, and X. Chou, Journal of Physics A: Mathematical and General 26, 1001 (1993).

19 A. Calogeracos and N. Dombey, Contemp. Phys. 40, 313 (1999).

${ }^{20}$ C. W. J. Beenakker, Rev. Mod. Phys. 80, 1337 (2008).

21 J. M. Pereira Jr, F. M. Peeters, A. Chaves, and G. A. Farias, Semiconductor Science and Technology 25, 033002 (2010). 
${ }^{22}$ R. V. Gorbachev, A. S. Mayorov, A. K. Savchenko, D. W. Horsell, and, F. Guinea, Nano Letters 8, 1995 (2008).

23 A. F. Young, and P. Kim, Nature Physics 5, 222 (2009).

24 A. F. Young, and P. Kim, Physical Review Letters 102, 026807 (2009).

25 B. Huard, J. A. Sulpizio, N. Stander, K. Todd, B. Yang, and D. Goldhaber-Gordon, Physical Review Letters 98, 236803 (2007).

26 A. K. Geim and K. S. Novoselov, Nature Materials 6, 183 (2007).

27 F. Guinea, M. I. Katsnelson, and A. K. Geim, Nature Physics 6, 30 (2010).

${ }^{28}$ V. M. Pereira, A. H. Castro Neto, H. Y. Liang, and L. Mahadevan, Phys. Rev. Lett. 105, 156603 (2010).

29 P. G. Silvestrov and K. B. Efetov, Phys. Rev. Lett. 98, 016802 (2007).

30 A. de Martino, L. Dell Anna, and R. Egger, Phys. Rev. Lett. 98, 066802 (2007).

31 G. Giavaras, P. A. Maksym, and M. Roy, Journal of Physics Condensed Matter 21, 102201 (2009).

32 M. M. Fogler, F. Guinea, and M. I. Katsnelson, Phys. Rev. Lett. 101, 226804 (2008).

33 G. Giavaras and F. Nori, App. Phys. Lett. 97, 243106 (2010).

${ }^{34}$ G. Giavaras and F. Nori, Phys. Rev. B 83, 165427 (2011).

35 A. K. Singh, E. S. Penev, and B. I. Yakobson, ACS Nano 4, 3510 (2010).

36 J.-A. Yan, L. Xian, and M. Y. Chou, Phys. Rev. Lett. 103, 086802 (2009).

37 S.-H. Cheng, K. Zou, F. Okino, H. R. Gutierrez, A. Gupta, N. Shen, P. C. Eklund, J. O. Sofo, and J. Zhu, Phys. Rev. B 81, 205435 (2010).

${ }^{38}$ H. J. Xiang, E. J. Kan, S.-H. Wei, X. G. Gong, and M.-H. Whangbo, Phys. Rev. B 82, 165425 (2010).

39 O. Oliveira, C. E. Cordeiro, A. Delfino, W. de Paula, and T. Frederico, Phys. Rev. B 83, 155419 (2011).

40 R. Jackiw, S.-Y. Pi, Phys. Rev. Lett. 98, 266402 (2007).

41 C.-Y.Hou, C. Chamon, and C. Mudry, Phys. Rev. Lett. 98, 186809 (2007).

42 C. Cordeiro, A. Delfino, and T. Frederico, Carbon 47, 690 (2009).

43 A. J. Chaves, G. D. Lima, W. de Paula, C. E. Cordeiro, A. Delfino, T. Frederico, O. Oliveira, Phys. Rev. B 83, 153405 (2011).

44 J. Gonzalez, F. Guinea, and M. A. Vozmediano, Phys. Rev. B 63, 134421 (2001). 